\title{
Euler Spring Collision Protection for Flying Robots
}

\author{
Adam Klaptocz, Adrien Briod, Ludovic Daler, Jean-Christophe Zufferey and Dario Floreano
}

\begin{abstract}
This paper addresses the problem of adequately protecting flying robots from damage resulting from collisions that may occur when exploring constrained and cluttered environments. A method for designing protective structures to meet the specific constraints of flying systems is presented and applied to the protection of a small coaxial hovering platform. Protective structures in the form of Euler springs in a tetrahedral configuration are designed and optimised to elastically absorb the energy of an impact while simultaneously minimizing the forces acting on the robot's stiff inner frame. These protective structures are integrated into a $282 \mathrm{~g}$ hovering platform and shown to consistently withstand dozens of collisions undamaged.
\end{abstract}

\section{INTRODUCTION}

Flying robots have the unique advantage of being able to provide human operators with an elevated viewpoint of places otherwise inaccessible to people. They are especially useful for the exploration of hard-to-reach places such as damaged buildings, irradiated nuclear power plants and underground mines that ground-based robots have trouble navigating because of clutter on the ground. The presence of a large amount of obstacles, however, along with lack of positioning and low visibility, make obstacle avoidance difficult and collisions with obstacles inevitable. Flying systems thus require protective structures if they are to survive in such cluttered environments.

In this paper we address the challenge of protecting a flying robot from the high impact energy of contact with obstacles or the ground. Rotor-based hovering platforms require a stiff inner frame, as their aerodynamics depend on the geometric positions and angles between rotors, the centre of gravity (COG) and/or control surfaces. Perhaps more importantly, flight motors cannot be allowed to flex out of plane in relation to the robots frame to prevent fast-spinning rotors from contacting other parts of the platform. The main requirement of protective structures on a flying robot is thus to shield this frame and the sensitive components mounted on it from damage resulting from multiple collisions.

Many current platforms use stiff protection mechanisms attached to an equally stiff inner frame to absorb collision energy through compression of the material [1], [2]. Though simple to design, stiff protection transfers all the impact energy of a collision to the platform's frame. As the absorption distance is minimal, the force on the frame quickly reaches high values. In the case of carbon fibre, the most

A. Klaptocz (corresponding author) and J.-C. Zufferey are with senseFly Ltd (http://www.sensefly.com), CH1024 Ecublens, Switzerland (email: adam.klaptocz@gmail.com). A. Briod, L. Daler, and D. Floreano are with the Laboratory of Intelligent Systems (http://lis.epfl.ch) at the Swiss Federal Institute of Technology in Lausanne (EPFL), CH1015 Lausanne, Switzerland. commonly used material for the frame of current platforms, impact damage is directly related to the impact force on a structure [3]. A single collision can cause extensive delamination, matrix shear cracking [4] and reduce the residual strength of the material [5], making it more vulnerable to subsequent collisions.

Impact force on the inner frame can be reduced by increasing the distance in which collision energy is absorbed. Some flying systems, such as the commercially-available AR.Drone[6], use foam rings around their rotors. This solution works well for low-energy collisions, but is too heavy to be dimensioned for high-energy impacts on flying systems due to its low specific stiffness. Another common protective structure is the spherical cage, often made of carbon fibre rods[7]. Such structures can absorb more energy for a given weight than foam but suffer from two major disadvantages; First, they are typically made of straight rods bent into a circular shape, store significant energy in the structure that cannot be used for collision energy absorption. Second, they are difficult to mount around the inner frame without using stiff connection points that can fail in a collision.

This paper aims at formalizing the design of protective structures for absorbing collision energy by presenting a method optimized for the weight constraints of flying robots. The method is applied to the protection of a small coaxial platform, yielding novel protective structures based on Euler springs arranged in a tetrahedral configuration. A prototype is built and a series of collision tests is performed to gauge the protective structures' performance. The paper is then concluded with a discussion of the results and future outlook.

\section{METHOD}

An ideal protective structure should limit the maximum force transmitted to the platform's stiff inner frame during a collision while minimizing its own weight. We propose the following steps to design and dimension protective structures adapted to flying platforms:

- Spring Type Selection and configuration: The type of spring and its configuration on the platform should be selected to increase absorption distance, minimize force on the platform's inner frame and protect from impacts in the most likely directions.

- Material Selection: A material should be chosen that has the required stiffness, yield strength and density to absorb the required energy.

- Dimensioning: Individual elements should be optimized to minimize weight. 
A

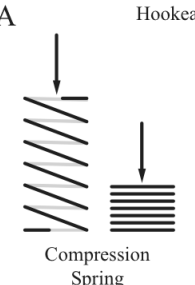

Hookean Spring
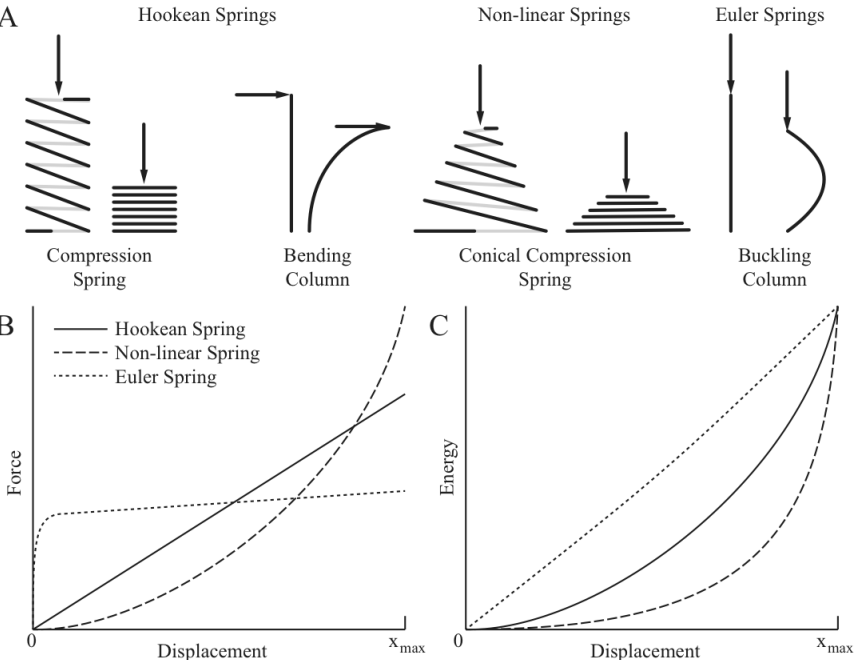

Fig. 1. (A) Some examples of the three common types of springs. Note that a column must be loaded axially to act in its buckling mode, otherwise in bending mode it acts as a Hookean spring. (B) A representative schematic of relative force and (C) energy profiles for the three types of springs, scaled for an equivalent amount of energy absorbed at maximum displacement.

\section{A. Spring Type Selection and Configuration}

A spring working in its elastic range absorbs energy $U$ over a distance $x_{\max }$ according to the formula:

$$
U\left(x_{\max }\right)=\int_{0}^{x_{\max }} F(x) \mathrm{d} x .
$$

where $F(x)$ is the force provided by the spring at displacement $x$. The total energy a spring can absorb is the area under the curve of $F(x)$ at the spring's maximum deflection, or the integration of Eq. 1. An ideal protective structure should thus limit the maximum force by increasing the absorption distance $x_{\max }$ and optimizing the the force profile $F(x)$.

There are three common types of springs: traditional linear or 'hookean' springs, non-linear springs (generally made of two or more different linear springs in series) and Eulermode buckling springs (Euler springs) (Fig. 1A). The force and energy profiles of these springs are shown in Fig. 1B and $\mathrm{C}$. Euler springs are long columns that are loaded along their axis to the point at which they buckle. Euler springs have the lowest maximum force exerted on their attachment point to a frame for an equivalent amount of energy and displacement and are thus a strong candidate for protective structure design.

Attaching a single Euler spring to the fuselage without fixing the other end will result in the spring slipping on the contact surface and bending (thus acting as a hookean spring) instead of buckling (acting as an Euler spring) (Fig 1A). To fix the end of the spring in place in three dimensions we propose using three Euler springs in a tetrahedral configuration as shown in Fig. 2A. The springs are attached to the fuselage and to each other using rotating joints to ensure that they are only loaded axially. If we model a rotor-based platform as a cylinder we can protect the platform from collisions in all directions by using eight tetrahedrals placed symmetrically around the platform.

The length of the individual spring elements is critical, as it defines the direction of the force loading during impact. If the elements are too short, an impact will bend them inwards, no longer in the direction of the force (Fig. 2B) and thus not absorbing energy through buckling. Conversely, if the elements are too long an impact bends the elements outwards (Fig. 2C). There is thus an optimized length of spring element that keeps the impact force close to the axial direction of the spring (Fig. 2D). In the case of eight tetrahedrals used symmetrically around a platform this formula can be derived through trigonometry (as shown in Fig. 2E and F):

$$
y=\sqrt{\frac{8}{3}} x
$$

where $y$ is the length of the spring and $x$ is the radius of the platform.

\section{B. Material Selection}

The material used for the spring should absorb the largest amount of energy without breaking (or plastic deformation) at the lowest weight. The three factors that must be considered are thus the material's stiffness (higher stiffness absorbs more energy) defined by its Young's modulus $E$, tensile yield strength $\mu$ (higher strength resists plastic deformation), and density $\rho$ (lower density yields lower weight). Materials can be optimized for weight by dividing the first two factors by their density, yielding Specific Stiffness and Specific Yield Strength, respectively. Some important material properties can be seen in Table I.

The material most adapted for use in flying robots is carbon-fibre reinforced plastic (or simply carbon fibre), as it has a specific stiffness more than $3 x$ higher than the stiffest metals. Pulltruded columns have slightly different characteristics than woven and stacked carbon fibre plates, and thus we tested some samples to extract their relevant properties. Deflection tests were performed using a linear stage and a load cell to measure the axial loading force required to bend carbon fibre columns of varying length and cross-sectional profiles in their buckling mode. The extracted material properties are added to Table I. The measured spring factor of a pulltruded rod in its linear mode (after buckling) is $k=8.09$.

\section{Dimensioning}

The goal of dimensioning is to select the lightest possible springs to absorb the desired energy without failing. There is generally a compromise to be made between the force transferred to the robot's frame, the weight of the spring and the amount of energy that it can absorb before failure, as well as secondary effects such as available materials, platform dimensions and integration. After selecting a spring type and configuration the first part of dimensioning is to determine the force profile of the protective structure independent of the second moment of inertia of the spring. The exact dimensions 


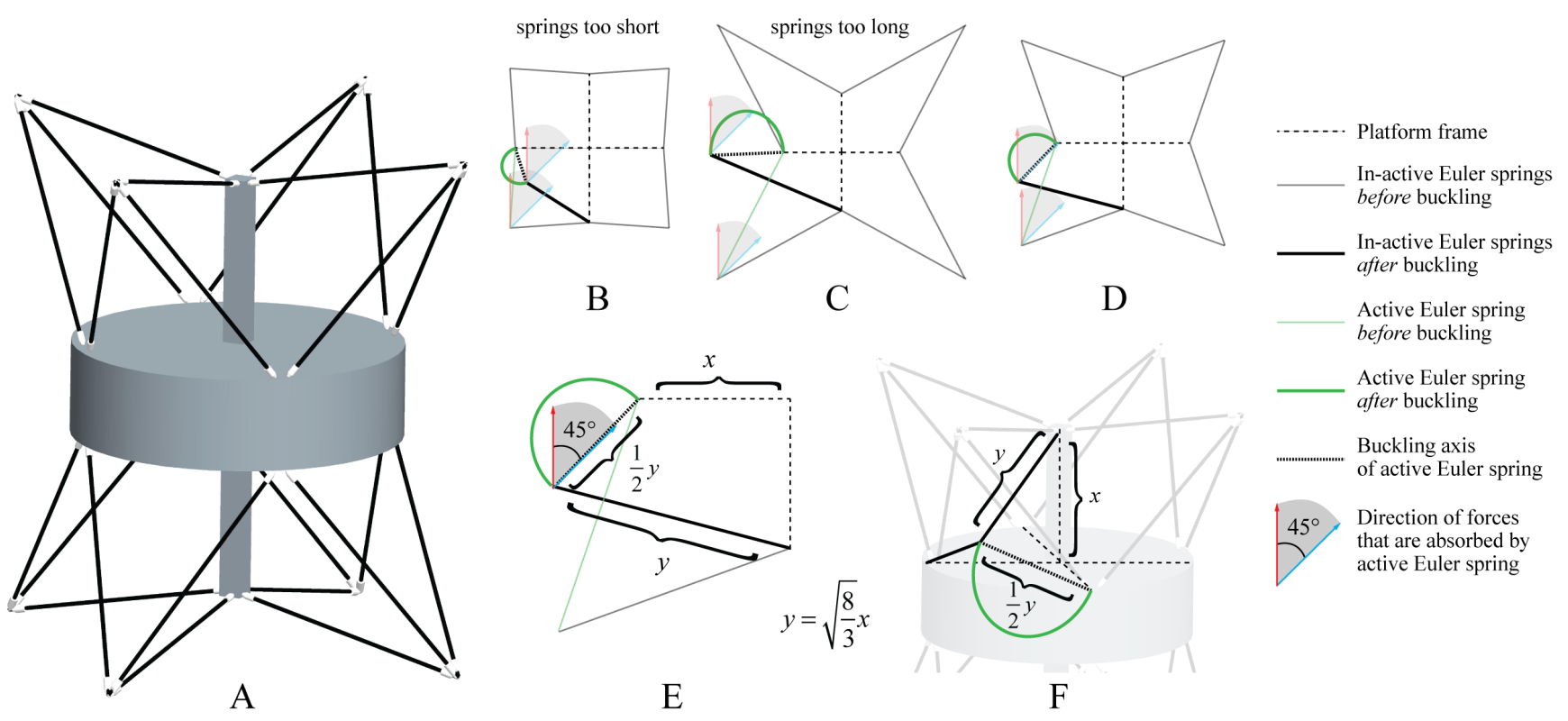

Fig. 2. (A) A proposed configuration of 8 tetrahedral structures consisting of three Euler springs for protecting a hovering platform. The length of the Euler springs is important, as it determines the amount of force that is applied in the axis of the spring during compression. The effect of spring length is illustrated in two dimensions in (B)-(D). The area between the red and blue force arrows show the range of force directions that must be absorbed by the green bending spring. (B) A spring that is too short bends inward, whereas (C) a spring that is too long bends outward. (D) An optimal length results in the force being directed near the axis of the spring throughout its compression. (E) The optimal length $y$ can be determined in the two-dimensional case based on the length of the base $x$ using trigonometry. (F) The same trigonometric calculation can be extended to three dimensions.

\begin{tabular}{|c|c|c|c|c|c|}
\hline Material & $\begin{array}{l}\text { Young's Modulus } \\
(G P a)\end{array}$ & $\begin{array}{lr}\text { Tensile } & \text { Yield } \\
\text { Strength }(M P a)\end{array}$ & $\begin{array}{l}\text { Density } \\
\left(\mathrm{g} / \mathrm{cm}^{3}\right)\end{array}$ & $\begin{array}{l}\text { Specific Stiffness } \\
(M N m / k g)\end{array}$ & $\begin{array}{l}\text { Specific Yield } \\
\text { Strength } \\
(k N m / k g)\end{array}$ \\
\hline Rubber (small strain) & $0.055+/-0.045$ & $8 *$ & $1.055+/-0.145$ & 0.0521 & 7.5 \\
\hline Brass & $112.5+/-12.5$ & 247 & $8.560+/-0.165$ & 13.14 & 29 \\
\hline Aluminum & 69 & $275+/-35$ & 2.700 & 25.56 & 102 \\
\hline Stainless Steel & 200 & 600 & $7.900+/-0.150$ & 25.32 & 76 \\
\hline Titanium alloy & $112.5+/-7.5$ & 977 & 4.510 & 24.94 & 217 \\
\hline Glass-Fibre-Reinforced Plastic (GFRP) & $31.65+/-14.45$ & $1500 *$ & 1.800 & 17.58 & 833 \\
\hline Aramid (e.g. Kevlar) & 70.5 & $2757 *$ & 1.440 & 48.96 & 1915 \\
\hline Carbon-Fibre-Reinforced Plastic (CFRP) & $135+/-15$ & $2000 *$ & 1.570 & 85.99 & 1273 \\
\hline Diamond & 1220 & 2800 & 3.530 & 345.61 & 793 \\
\hline Pulltruded Carbon Fiber Columns & $113+/-15$ & $2992+/-700$ & 1.47 & 76 & 2035 \\
\hline
\end{tabular}

TABLE I

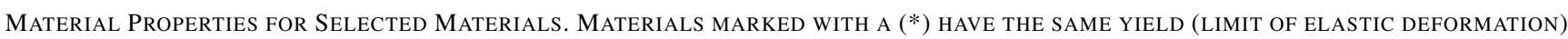

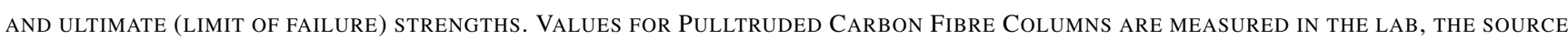
OF OTHER MATERIAL DATA IS HTTP://WWW.MATWEB.COM/. THESE ARE JUST INDICATIVE FIGURES, AS THEY CAN VARY GREATLY DEPENDING ON SAMPLES AND TESTING METHODS.

of the spring elements are then selected based on the required energy to be absorbed, material properties and weight.

As we are using pulltruded carbon fibre rods whose length is determined by the platforms dimensions according to Eq. 2 the problem is reduced to dimensioning the radius $r$ of rod to be used that absorbs the required energy without failure while minimizing weight. The balance between amount of energy absorbed, the force on the platforms frame and the weight will depend on the constraints of the platform. A particular implementation is demonstrated in the following section.

\section{PROOF-OF-CONCEPT}

The protective structure design method above is applied to the protection of a small flying robot similar to the one previously published [8], [9]. The core of the platform is defined by a coaxial motor with $10 \mathrm{~cm}$-diameter rotors. Allowing for protection around the rotors, the platforms stiff internal frame takes a cylindrical shape with a radius of $12 \mathrm{~cm}$. Using Eq. 2 the optimal length of the Euler springs is aprox. $20 \mathrm{~cm}$. To dimension the radius of rod required we use Eqs. 6, 7, 11, 12, 16, derived in the appendix using standard beam theory, to get a sense of the amount of energy we can expect to absorb. 

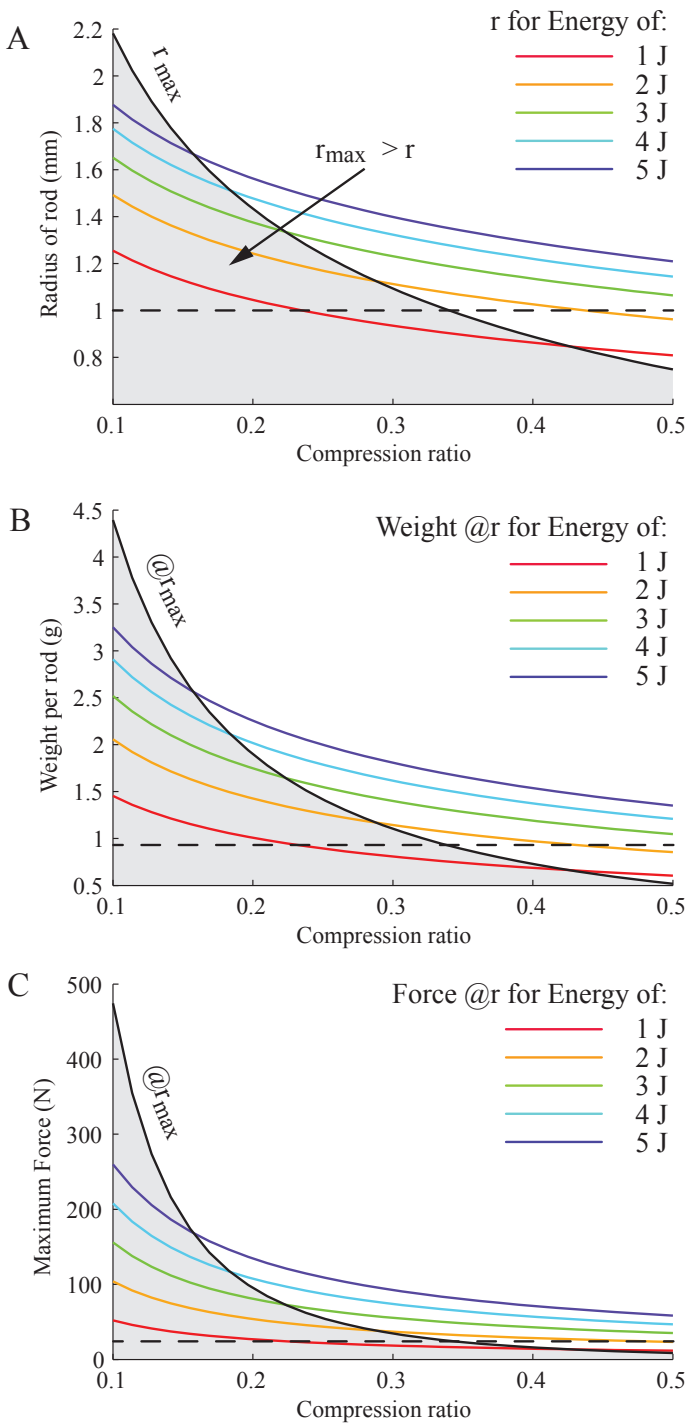

Fig. 3. (A) Rod radius, (B) weight and (C) maximum force for a $20 \mathrm{~cm}$ carbon fibre rod as an euler spring. Shaded areas represent possible combinations of compression ratio, radius and energy for which the rod will not fail.

Figure 3 plots the radius, weight and force of a $20 \mathrm{~cm}$ rod as a function of compression ratio (the ratio between the lengths of the compressed and uncompressed spring). As a general trend, maximizing the compression ratio will lower both the force on the inner frame and the weight of the rod. There is a fixed maximum radius however which decreases with the compression ratio, since a thicker rod will fail at a lower bend angle than a thinner one. As this robot is designed to fly indoors (low altitude, slow speeds) it should not encounter high-energy impacts and thus we optimize for minimal weight and force rather than maximum energy. We select a rod radius of $1 \mathrm{~mm}$ (dashed line in Fig. 3), which will allow the platform to absorb $1.5 \mathrm{~J}$ at a compression ratio of 0.34 . With an estimated platform weight of $300 \mathrm{~g}$ (based on the weight of previous systems), this is the energy of a freefall from a height of $51 \mathrm{~cm}$. It should be noted that this is the energy that can be absorbed by a single rod. As there are 24 rods surrounding the platform, in most cases there will be several rods that will absorb the energy at once.

Figure 4D shows the completed flying platform including protective structures attached to a rigid internal frame. The frame houses flight motors, control surfaces and electronics (Fig. 4A). The carbon-fibre Euler springs are inserted into end pieces made of laser-sintered ABS plastic which are subsequently attached to the frame (Fig. 4B) and to each other (Fig. 4C) using nylon fishing line. The weight of the protective structures including end pieces is $40 \mathrm{~g}$, around $14 \%$ of the of the platform's $282 \mathrm{~g}$ total weight.

\section{RESUlts}

The platform with its protective structures was put through a series of tests to evaluate its robustness to contact. Impact force on the frame was measured using 3 orthogonally-positioned single-axis high-G accelerometers (Freescale MMA3204, range of +/- 100 G, Fig. 4A) coupled directly to the platforms frame. Depending on the direction of the impact vector, the total acceleration that can be measured ranges from $100-173 \mathrm{G}$ or $980-1695 \mathrm{~m} / \mathrm{s}^{2}$. With a platform mass of $282 \mathrm{~g}$ this corresponds to a maximum measurable force of $276-478 \mathrm{~N}$.

\section{A. Buckling Motion Validation}

The goal of the first experiment is to validate that the Euler springs are indeed buckling during a collision. The platform is dropped from a height of $1 \mathrm{~m}, 5$ times on its base (the position most likely to occur during a hard landing) and 5 times on a single tetrahedral protective structure, and filmed at 1000 fps using a high-speed camera. All the videos are subsequently analyzed, confirming that in each case one or more Euler springs is buckling to absorb impact energy. Figure 5A shows a timeseries of an impact on the platform's base in which four Euler springs, one from each tetrahedral, are buckling concurrently. In Fig. 5B a single Euler spring absorbs impact energy and subsequently releases it, causing the platform to spin. The remaining energy is then absorbed by two Euler springs of a different tetrahedral.

\section{B. Impact Force Minimization}

The goal of this experiment is to compare the force minimization capability of the Euler-spring protective structures to its stiff and foam-based counterparts. For this comparison a styrofoam protective structure with the same weight as the Euler spring structures is built for the platform and is shown in Fig. 4E. The platform's bare inner frame is used as the stiff protective structure (Fig. 4F). The platform is dropped 5 times from increasing heights with either the stiff, the Euler spring or the styrofoam protective structures, first on its base and then on a corner (vertex of a tetrahedral for Euler spring structures, between the propeller-protecting ring and two sides for stiff or styrofoam structures). The height is increased until the high- $\mathrm{G}$ accelerometers saturate.

Figure 6A shows a box plot of the force measured on the platform for each experiment whereas Fig. $6 \mathrm{~B}$ shows the 

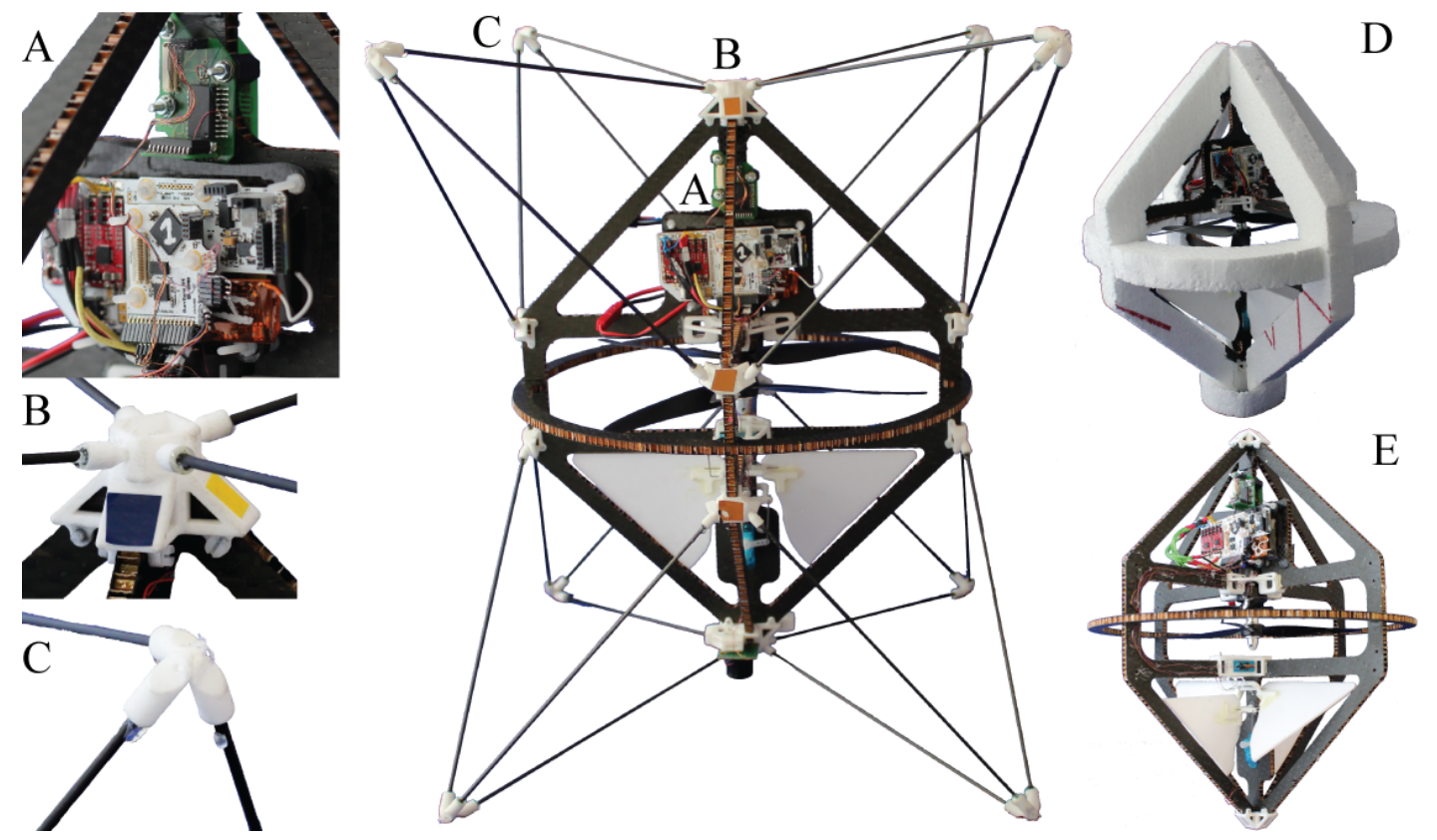

Fig. 4. (D) The hovering flying robot equipped with Euler-spring protection structures. (A) shows the on-board electronics, including high-G accelerometers (top circuit board) mounted directly to the robot's frame whereas (B) and (C) show the two endpoints of the Euler springs. The same core frame is outfitted with (E) styrofoam and $(\mathrm{F})$ stiff protection for force comparison experiments.

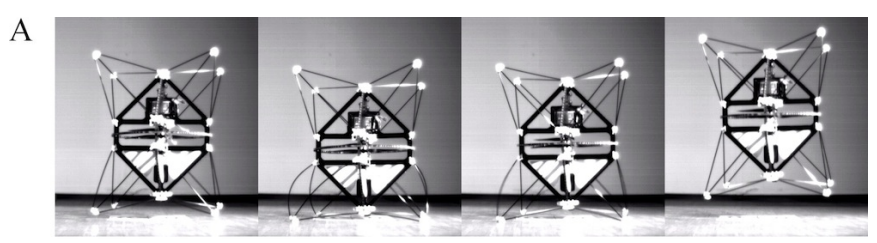

$\mathrm{B}$

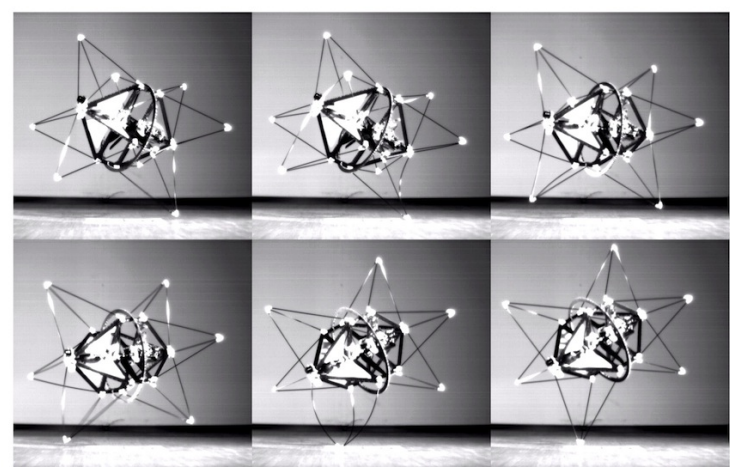

Fig. 5. Timeseries of drop tests on (A) the platforms base and (B) on a single tetrahedral from a height of $75 \mathrm{~cm}$.

profile of the force through time for a drop height of $75 \mathrm{~cm}$. The following conclusions can be derived from these plots:

- Using only stiff protection of rotors results in high impact forces even at a height of $10 \mathrm{~cm}$.

- When falling on its base, the Euler spring mechanism has higher initial forces than the foam mechanism. This is due to the initial buckling force $F_{\text {crit }}$ from 4 contact points that is reached before any energy is absorbed. Once this force is reached, however, it does not increase significantly even at $75 \mathrm{~cm}$ in accordance with the nearflat force profile of Euler springs.
- Foam-based protection transfers significantly more force to the platform's frame at high drop heights.

- Force profiles show that Euler springs absorb energy over a longer time period, thus decreasing the force on the frame.

As a general conclusion Euler spring protection mechanisms perform similarly to foam-based mechanisms at low heights, but significantly decrease impact force on the robot's frame in high-energy impacts. These trends could be better observed with higher drop heights but were not done for two reasons: accelerometers with higher thresholds were not available, and more importantly subjecting the stiff internal frame to higher forces would risk breaking the frame, making subsequent tests impossible.

\section{Resilience}

The robot is placed in the centre of a $3.5 \times 6 \mathrm{~m}$ experimentation room. A simple behaviour is programmed in the onboard micro-controller which makes the platform take off, stabilize at a hovering altitude of $1 \mathrm{~m}$ for $3 \mathrm{~s}$, then move in a random direction until it collides with a wall. Once a collision is detected with the on-board accelerometers and gyroscopes the motors are cut and the platform allowed to fall to the ground.

The experiment is repeated 50 times to simulate collisions from many directions with varying amounts of impact energy. From the 50 trials, only 4 collisions resulted in damage to the platform: $2 \mathrm{x}$ the soft rotors flexed and touched each other and $2 \mathrm{x}$ the nylon strings connecting end pieces failed. Using stiffer rotors and increasing the thickness of the nylon string should limit these failure modes. This experiment demonstrates the robustness of the protective 

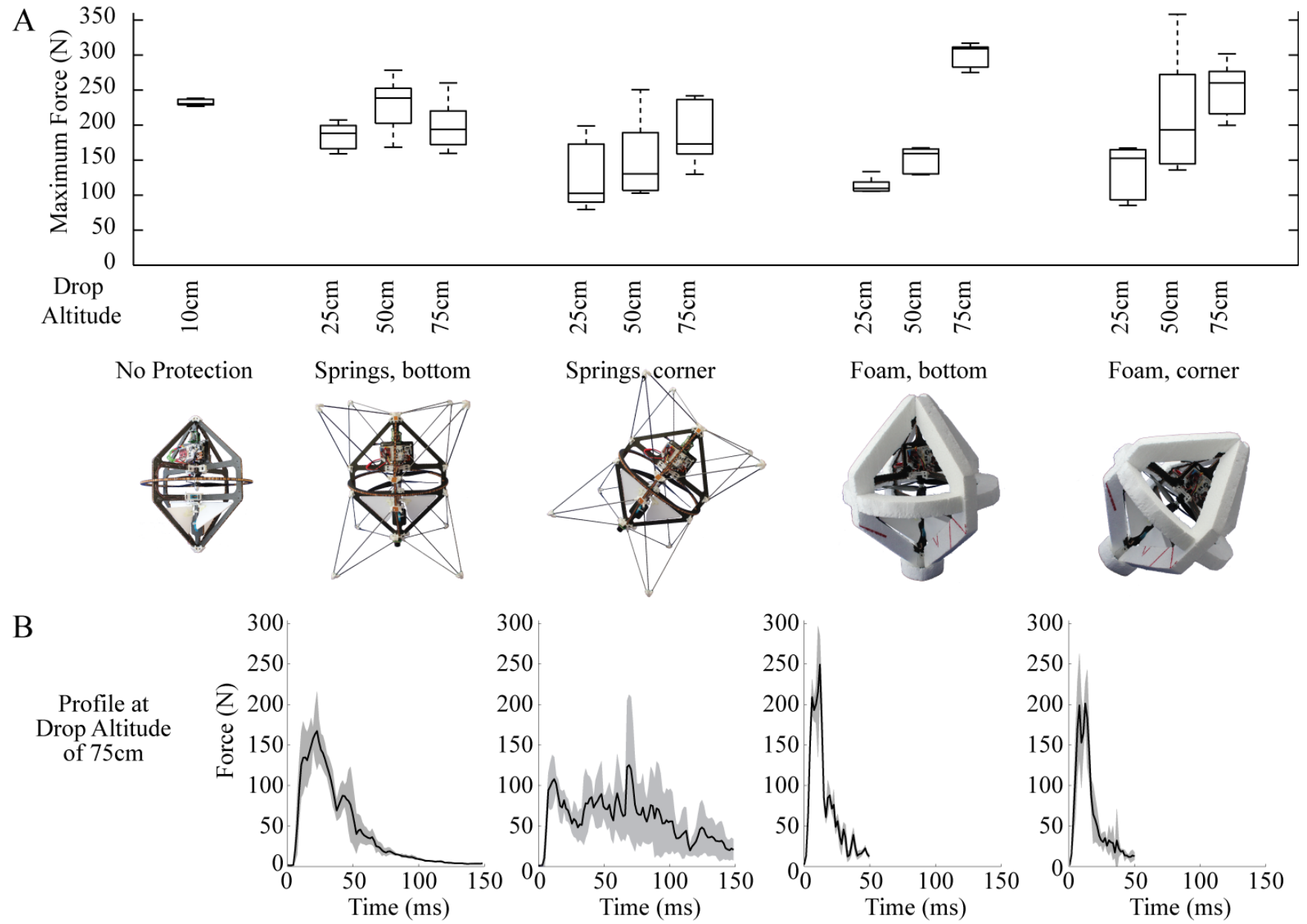

Fig. 6. (A) boxplot of the maximum force measured on the platforms frame over 5 trials for drop tests from varying altitudes and using various protection mechanisms. (B) Force profile over time averaged over 5 trials for a free fall from $75 \mathrm{~cm}$. Shaded regions represent standard deviation. As only a limited amount of data points can be sent to the ground station, and initial tests showed a flat force curve after $50 \mathrm{~ms}$ using foam protection, it was decided to only record data from the first $50 \mathrm{~ms}$ to increase temporal resolution.

structures to repeated high-energy collisions. A sample of the many collisions can be seen in the accompanying video.

\section{Conclusions}

This paper presents a novel protection structure design using Euler springs optimized for flying robots that must survive repeated high-energy impacts with their environment. The structure is shown to greatly reduce the impact forces transferred to a robot's frame compared to existing solutions using stiff or foam-based protection. The design is adapted to a small hovering platform and validated through an exhaustive amount of static drop tests and collision tests during flight in an indoor environment.

The ability to absorb impact energy elastically allows flying robots to access constrained and cluttered environments that were previously unattainable. It also opens the door to new behaviors for exploration such as contact-based navigation, already used by ground robots when low light or smoke render vision or laser-based navigation impossible.

\section{APPENDIX}

According to standard beam theory [10], a column will buckle when it reaches critical force $F_{\text {crit }}$ :

$$
F_{\text {crit }}=\frac{\pi^{2} E I}{L^{2}}
$$

where $L$ is the column's length, $E$ is the Young's modulus of the material and $I$ is the column's area moment of inertia. The force profile of an Euler buckling spring $F(x)$ is:

$$
\begin{aligned}
F(x) & =F_{\text {crit }}+\frac{E I}{L^{2}} k \frac{x}{L} \\
& =\frac{E I}{L^{2}}\left(\pi^{2}+k \frac{x}{L}\right)
\end{aligned}
$$

where $k$ is the spring factor and $0<x<L$. We can normalize Eq. 4 for a given compression factor $c$ by substituting the following equation:

$$
c=\frac{x}{L}
$$

(where $0<c<1$ ) which results in: 


$$
F(c)=\frac{E I}{L^{2}}\left(\pi^{2}+k c\right)
$$

We can calculate the energy absorbed by a column by substituting Eq. 6 into Eq. 1:

$$
\begin{aligned}
U & =L \int F(c) \mathrm{d} c \\
& =\frac{E I c}{L}\left(\pi^{2}+\frac{1}{2} k c\right)
\end{aligned}
$$

Solving for $I$ we can compute the second moment of inertia $I$ required to absorb this energy:

$$
I=\frac{U L}{E c} \frac{1}{\pi^{2}+\frac{1}{2} k c}
$$

For a cylindrical rod with radius $r$,

$$
I=\frac{\pi r^{4}}{4}
$$

Rearranging for $r$ :

$$
r=\sqrt[4]{\frac{4 I}{\pi}}
$$

Substituting Eq. 8 into Eq. 10 and we can write in terms of $L, c$ and $U$ :

$$
r=\sqrt[4]{\frac{4 U L}{\pi E c} \frac{1}{\pi^{2}+\frac{1}{2} k c}}
$$

The upper limit of allowable rod radius $r_{\max }$ depends on the strain that the rod can take before breaking. For this we calculate the maximum force at the desired compression ratio:

$$
r_{\max }=\frac{\mu I}{F_{\max } y}
$$

where $F_{\max }$ is the force at which the column fails and $y$ is the displacement of the rod perpendicular to its axis at $F_{\max }$. We can estimate $y$ with good accuracy using the following formula [11]:

$$
y=0.900 L\left(\frac{F_{\max }}{F_{\text {crit }}}-1\right)^{\frac{1}{2}}
$$

Substituting Eq. 13 into Eq. 12 and writing once again in terms of $L, c$ and $U$ we have:

$$
r_{\max }=\frac{\mu L}{0.9 E} \frac{\pi}{\left(\pi^{2}+k c\right) \sqrt{k c}}
$$

In order to absorb the desired energy $U$ without breaking there must exist values of $L$ and $c$ for which $r<r_{\max }$.

Finally, the weight of the rod can be computed using its volume and density:

$$
W_{\text {rod }}=\rho \pi r^{2} L
$$

The minimum weight $W_{\min }$ at $r$ in terms of $L$ and $c$ can be derived by substituting Eq. 11 into Eq. 15:

$$
\begin{aligned}
W_{\min }= & \rho \pi r^{2} L \\
= & \rho \pi \sqrt{\frac{4 U L}{\pi E c} \frac{1}{\pi^{2}+\frac{1}{2} k c}} \\
& \times \sqrt[3]{\frac{0.9^{4} 4 E^{3} U\left(\pi^{2}+k c\right)^{4}(k c)^{2}}{\mu^{4} c \pi^{5}\left(\pi^{2}+\frac{1}{2} k c\right)}}
\end{aligned}
$$

ACKNOWLEDGMENTS

This work was supported by the Swiss National Science Foundation through the National Centre of Competence in Research Robotics.

\section{REFERENCES}

[1] G. Wang, H. Sheng, T. Lu, D. Wang, and F. Hu, "Development of an Autonomous Flight Control System for Small Size Unmanned Helicopter *," in IEEE International Conference on Robotics and Biomimetics, 2007, pp. 1804-1809.

[2] M. Itasse and J.-M. Moschetta, "Equilibrium Transition Study for a Hybrid MAV," International Journal of Micro Air Vehicles, vol. 3, no. 4, pp. 229-245, 2011.

[3] A. Christoforou, "Impact dynamics and damage in composite structures," Composite structures, vol. 52, 2001.

[4] G. Davies and X. Zhang, "Impact damage prediction in carbon composite structures," International Journal of Impact Engineering, vol. 16 , no. 1 , pp. 149-170, 1995.

[5] W. Cantwell, P. Curtis, and J. Morton, "Impact and subsequent fatigue damage growth in carbon fibre laminates," International journal of fatigue, vol. 6, no. 2, pp. 113-118, 1984.

[6] P.-J. Bristeau, F. Callou, D. Vissière, and N. Petit, "The Navigation and Control technology inside the AR.Drone micro UAV," in Internation Federation of Automatic Control (IFAC) World Congress, 2011.

[7] E. Beyer and M. Costello, "Performance of a hopping rotochute," International Journal of Micro Air Vehicles, vol. 1, no. 2, pp. 121-137, 2009.

[8] A. Klaptocz, G. Boutinard-Rouelle, A. Briod, J.-C. Zufferey, and D. Floreano, "An Indoor Flying Platform with Collision Robustness and Self-Recovery," in IEEE International Conference on Robotics and Automation. IEEE, 2010, pp. 3349-3354.

[9] A. Klaptocz, L. Daler, A. Briod, J.-C. Zufferey, and D. Floreano, "An Active Uprighting Mechanism for Flying Robots," IEEE Transactions on Robotics, 2012.

[10] W. C. Young and R. G. Budynas, Roarks Formulas for Stress and Strain, 2002.

[11] R. Frisch-Fay, Flexible Bars. London: Butterworths1962, 1962. 